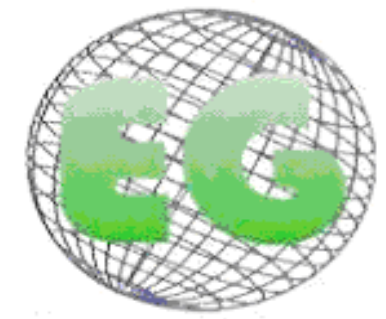

ISSN 1695-6141

N24

\title{
Técnicas de observación y la temática calidad de vida: una revisión integrativa
}

\author{
TÉCNICAS OBSERVACIONAIS E A TEMÁTICA QUALIIDADE DE VIIDA: UMA \\ REVISÃO INTEGRATIVA
"Zamberlán, C. ${ }^{* *}$ Calvetti, A., ${ }^{* *}$ Reinstein de Figueiredo, T., ${ }^{* *}$ Dei Svaldi, J., ${ }^{* * * * H e c k l e r ~ d e ~ S i q u e i r a, ~ H C . ~}$

\begin{abstract}
*Enfermera, Unidade de Cardiologia Intensiva (UCI), Hospital Universitário de Santa Maria (HUSM/UFSM). **Enfermera. Miembro del Grupo de Pesquisa GEES. *** Doctora en Enfermería por la FURG, Miembro del GEES.**** Doctora en Enfermería. Docente da Escola de Enfermagem de la FURG. Líder del Grupo de Estudos e Pesquisa: Gerenciamento Ecossistêmico em Enfermagem/Saúde. Brasil.
\end{abstract}

Palabras clave: Enfermería; Calidad de vida; Observación.

.Palavras-chave: Enfermagem; Qualidade de Vida; Observação

Keywords: Nursing; Quality of life; Note.

\section{RESUMEN}

En el contexto actual la calidad de vida puede percibirse desde varios puntos de vista. Esta calidad emerge de situaciones vividas y experimentadas por cada individuo. Este fenómeno está vinculado a la circularidad, la interdependencia e influencia que los elementos constituyentes de una zona, región o ambiente tienen entre sí. Se tuvo como objetivo describir las técnicas de observación que se utilizaron en la producción científica de enfermería que abordó la cuestión de calidad de vida. Investigación bibliográfica del tipo revisión integrativa.. El corpus de análisis fue de doce artículos. Como resultado, se puede ver la emergente necesidad de más investigación en enfermería sobre la calidad de vida mediante la técnica de observación teniendo en cuenta que el tema es ampliamente discutido en la actualidad en diferentes espacios.

\section{RESUMO}

No contexto atual a qualidade de vida pode ser percebida sob diversos enfoques. Esta qualidade emerge de situações vividas e experienciadas por cada indivíduo. Esse fenômeno encontra-se ligado à circularidade, a interdependência e influência que os elementos constituintes de um espaço/território/ambiente mantêm entre si. Teve-se como objetivo descrever as técnicas observacionais que foram utilizadas na produção científica da enfermagem que abordaram a temática qualidade de vida. Trata-se de pesquisa bibliográfica, do tipo revisão integrativa. O corpus de análise correspondeu a doze artigos. Como resultados, pode-se perceber, a 
necessidade emergente de mais pesquisas na enfermagem sobre qualidade de vida com utilização da técnica de observação tendo em vista que a temática é amplamente discutida na atualidade em diferentes espaços.

\section{ABSTRACT}

Today quality of life can be seen from several viewpoints. This quality emerges in situations lived and experienced by each individual. This phenomenon is connected to the circularity, interdependence and influence that the constituents of an area / region / environment have with each other. The objective was to describe the observational techniques that were used in nursing scientific production that addressed the quality of life issue. An integrative review of the literature was made. The corpus amounted to twelve articles. As a result, one can see, the emerging need for more research in nursing on quality of life using the technique of observation given that the subject is widely discussed nowadays in different spheres

\section{INTRODUCCIÓN}

En el contexto actual la calidad de vida puede ser percibida bajo distintos plantamientos. Esa calidad surge de situaciones vividas y experimentadas por cada individuo. Este fenómeno se encuentra ligado a la circularidad, la interdependencia e infuencia que los elementos constitutivos de un espacio/territorio/ambiente mantienen entre sí.

En esta perspectiva el espacio/ambiente cohabitado por los seres humanos suscita maneras de vivir con características propias y que pueden posibilitar la ocurrencia de una mejor o peor calidad de vida. Por lo tanto, hubo la necesidad de estudiar determinadas áreas del conocimiento para establecer respuestas confiables a la calidad de vida ya que tiene por definición uma dimensión compleja que sigue la dinámica de las poblaciones en sus distintas culturas, valores y creencias ${ }^{(1)}$.

La calidad de vida por medio de su característica integradora, interrelacional y multidimensional surge en los varios contextos de la salud y en las múltiples dimensiones humanas, como la biológica, la social, psicológica y social. Además, los aspectos de la calidad de vida se interrelacionan con el ambiente donde los sujetos están insertados y necesitan atención, para permitir el equilíbrio y sustentabilidad de todos aquellos que participan de esa totalidad ${ }^{(2)}$.

Mientras tanto, la observación del ambiente en que los sujetos viven y conviven puede propiciar la visualización de acciones que por medio de otras técnicas no sería posible. Así, la observación de alguna manera se dirige al encuentro de un problema y el investigador en este método acompaña personas y eventos para observar los comportamientos y relaciones cotidianos $^{(3)}$.

Basado en este pensamiento surge como problema de investigación: ¿Cómo son utilizadas las técnicas observacionales en las producciones científicas de enfermería que abordaron la temática de calidad de vida?

En este abordaje y al mismo tiempo en un contexto más amplio, la temática calidad de vida se relaciona con múltiplos espacios, a través de acciones y actividades que se construyen y reconstruyen de forma distinta, lo que contribuye al diálogo, la discusión y la construcción del conocimiento desde la perspectiva de la promoción de la salud ${ }^{(2)}$.

Por lo tanto, este estudio tuvo como objetivo describir las técnicas observacionales que fueron utilizadas en la producción científica de enfermería que abordaron la temática calidad de vida. 


\section{REVISIÓN INTEGRATIVA}

Este estudio se caracteriza como uma revisión integrativa (RI), método cuya finalidad es la sumarización de investigaciones ya hechas, para obtener nuevas conclusiones a partir de um tema de interés ${ }^{(4)}$.

Por reunir resultados de pesquisas sobre un tema delimitado o cuestión, de manera sistemática y ordenada, la RI contribuye a que el conocimiento del tema sea ampliado y, además, para que se obtenga un mejor entendimiento de un determinado fenómeno basándose en estudios anteriores ${ }^{(5)}$.

Para trabajar esta revisión se utilizaron las siguientes etapas: delimitación del objetivo; definición de los criterios de inclusión de las producciones científicas; búsqueda de los estudios en las bases de datos; análisis de los resúmenes de los estudios; selección de los estudios de acuerdo con los criterios de inclusión; evaluación crítica de los estudios y elección de los estudios seleccionados y análisis de los datos ${ }^{(4)}$.

El levantamiento bibliográfico fue realizado en todas las bases de datos de la Biblioteca Virtual en Salud, durante el mes de diciembre de 2010, con delimitación del período de publicación entre los años de 2001 y 2010 y utilizando las siguientes palabras clave: calidad de vida , enfermería y estudios observacionales.

Fueron utilizados como criterios de inclusión: artículos científicos publicados en revista brasileña indexados en la base Scientific Eletronic Library Online (SciELO) y disponibles online; período de 01 de enero de 2001 a 01 de diciembre de 2010; palabra calidad de vida u observación presente en el resumen; artículos presentando la observación como técnica de colecta de datos, que puede o no estar asociada a otra técnica y tener por lo mínimo, un autor enfermero.

Fue elaborado un instumento para colecta de datos designado formulario de registro abarcando el código de referencia, título de la publicación, autores, fuente de publicación, tipo de artículo, área de actuación de la enfermería en la cual la calidad de vida fue utilizada.

\section{RESULTADOS}

Por medio de la colecta de datos investigados surgieron 23 artículos, y de estos, uno fue publicado en el año 2001, uno en 2002, dos en 2003, dos fueron publicados en 2004, cinco en 2005, uno en 2006, ninguno en 2007, cinco en 2008, cuatro en 2009 y dos en 2010.

Fueron excluidos, por no presentar por lo mínimo un enfermero como autor: en 2001 un único artículo fue excluído, en 2003 uno, en 2006 un solo artículo fue excluído, en 2008 cuatro artículos, en 2009 tres y en 2010 un artículo.

Al terminar la colecta de datos, fueron selecionados 12 artículos, que se adecuaron a la investigación y por eso compusieron el corpus de análisis, como puede verse en la Tabla I. 
Tabla I - Artículos excluídos y utilizados sobre calidad de vida, utilizando la técnica de observación de acuerdo con los años de publicación 2001 - 2010, SciELO, Brasil, 2010.

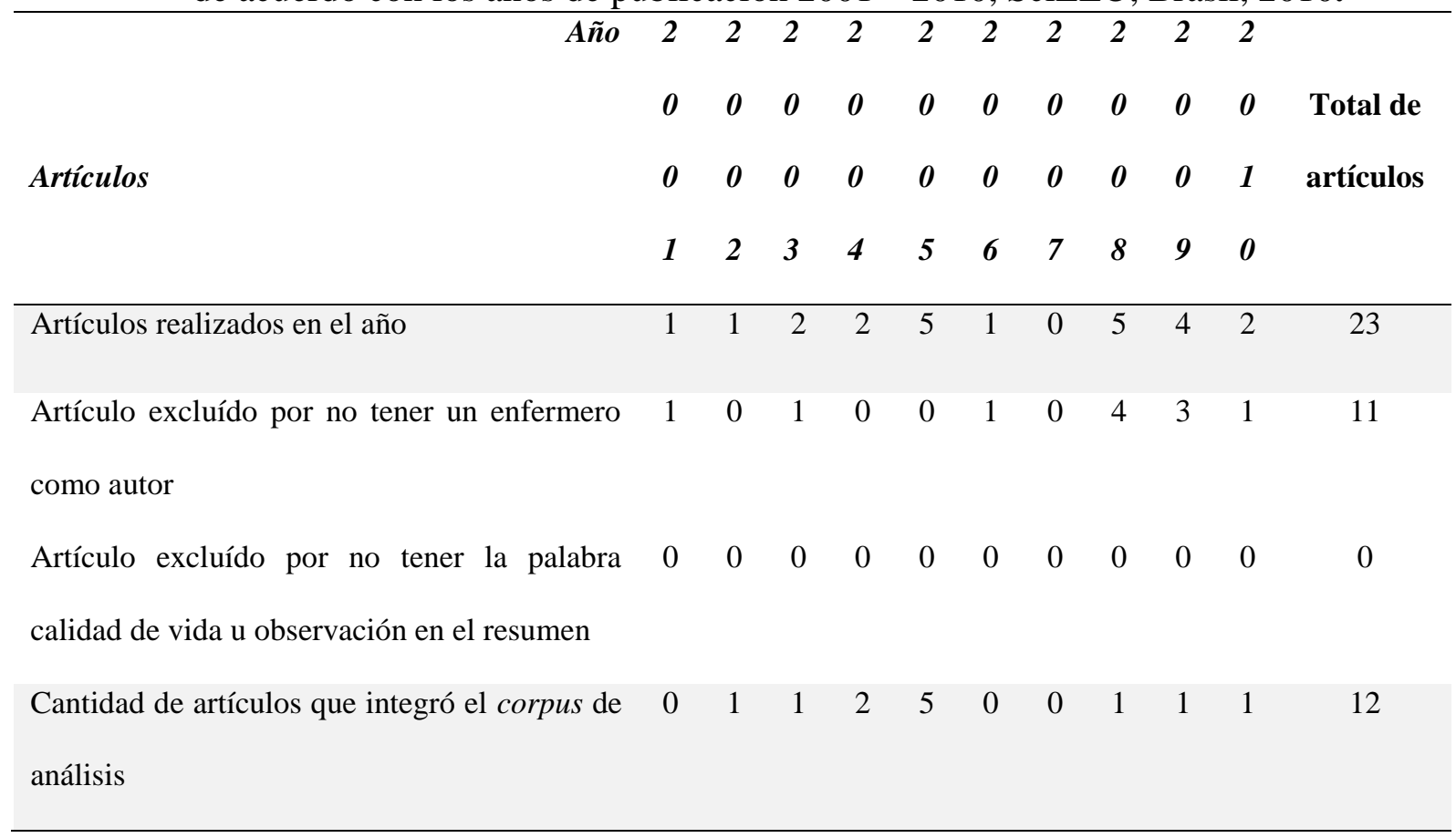

Se verificó que once artículos no tuvieron un enfermero como autor/coautor, demostrando que el tema calidad de vida con utilización de la técnica de observación como la colecta de datos es más abordado por profesionales de otras áreas (Educación, Sociología, Antropología, Psicología, Medicina y otras), confirmando la base de datos y tiempo pesquisado.

Otro descubrimiento importante fue que todos los artículos seleccionados citaron en el resumen las palabras clave calidad de vida y observación. A continuación se presenta la Tabla II, que demuestra las referencias de los materiales seleccionados, teniendo en cuenta los criterios de inclusión de los datos.

Tabla II - Descripción de las publicaciones según el código de referencia, título de la publicación, autor, fuente de publicación, formación del autor, año de publicación, tipo de artículo y estudio y metodologia utilizada. SciELO, Brasil, 2010.

\begin{tabular}{|c|c|c|c|c|c|c|c|}
\hline Cód & $\begin{array}{c}\text { Título de la } \\
\text { publicación }\end{array}$ & Autor & Fuente & $\begin{array}{c}\text { Formación } \\
\text { del autor }\end{array}$ & Año & $\begin{array}{c}\text { Tipo de } \\
\text { artículo y } \\
\text { estudio }\end{array}$ & $\begin{array}{c}\text { Metodología } \\
\text { utilizada }\end{array}$ \\
\hline 1 & $\begin{array}{c}\text { Apoio social e } \\
\text { redes: conectando } \\
\text { solidariedade e } \\
\text { saúde }\end{array}$ & $\begin{array}{c}\text { Andrade, } \\
\text { GRB; } \\
\text { Vaitsman,J }\end{array}$ & $\begin{array}{c}\text { Ciência e } \\
\text { Saúde coletiva }\end{array}$ & $\begin{array}{c}\text { Enfermero } \\
\text { Asistente } \\
\text { social }\end{array}$ & 2002 & $\begin{array}{c}\text { Pesquisa } \\
\text { Cualitativa }\end{array}$ & $\begin{array}{c}\text { Entrevista semi } \\
\text { estructurada y } \\
\text { observación } \\
\text { participante }\end{array}$ \\
\hline 2 & $\begin{array}{c}\text { Avaliação da } \\
\text { assistência pré-natal } \\
\text { oferecida em uma } \\
\text { microrregião de } \\
\text { saúde do Ceará, } \\
\text { Brasil }\end{array}$ & $\begin{array}{c}\text { Moura, ERF, } \\
\text { Holanda,F; } \\
\text { Rodrigues, } \\
\text { MSP }\end{array}$ & $\begin{array}{c}\text { Cadernos de } \\
\text { Saúde Pública }\end{array}$ & Enfermero & 2003 & $\begin{array}{c}\text { Pesquisa } \\
\text { documental } \\
\text { Cuantitativa }\end{array}$ & $\begin{array}{c}\text { Observación } \\
\text { libre y revisión } \\
\text { de prontuarios }\end{array}$ \\
\hline 3 & $\begin{array}{c}\text { Cesarianas: } \\
\text { percepção de risco e } \\
\text { sua indicação pelo } \\
\text { obstetra em uma } \\
\text { maternidade pública }\end{array}$ & $\begin{array}{c}\text { Dias, MAB; } \\
\text { Deslandes, } \\
\text { SF }\end{array}$ & $\begin{array}{c}\text { Cadernos de } \\
\text { saúde Pública }\end{array}$ & Médico \\
Enfermero & 2004 & $\begin{array}{c}\text { Pesquisa } \\
\text { Cualitativa }\end{array}$ & $\begin{array}{c}\text { Observación } \\
\text { participante } \\
\text { Entrevista }\end{array}$ \\
\hline
\end{tabular}




\begin{tabular}{|c|c|c|c|c|c|c|c|}
\hline & $\begin{array}{l}\text { no município do } \\
\text { Rio de Janeiro }\end{array}$ & & & & & & \\
\hline 4 & $\begin{array}{l}\text { Swedisch advanced } \\
\text { home care: } \\
\text { organizational } \\
\text { structure and } \\
\text { implications of } \\
\text { adopting this care } \\
\text { model in brazilian } \\
\text { health care system }\end{array}$ & $\begin{array}{l}\text { Perroca, MP, } \\
\text { EK, AC }\end{array}$ & $\begin{array}{l}\text { Revista latino } \\
\text { Americana de } \\
\text { enfermagem }\end{array}$ & Enfermero & 2004 & $\begin{array}{l}\text { Pesquisa } \\
\text { Cualitativa }\end{array}$ & $\begin{array}{l}\text { Entrevista } \\
\text { Cuestionario } \\
\text { Observación }\end{array}$ \\
\hline 5 & $\begin{array}{l}\text { Promoción de la } \\
\text { salud Y calidad de } \\
\text { vida entre madres } \\
\text { de preadolescentes; } \\
\text { uma etnografia } \\
\text { enfocada } \\
\end{array}$ & $\begin{array}{l}\text { Luduena, } \\
\text { AC; Olson, } \\
\text { JK; Pasco, } \\
\text { ACY }\end{array}$ & $\begin{array}{l}\text { Revista latino } \\
\text { AM Enferma- } \\
\text { gem }\end{array}$ & Enfermero & 2005 & $\begin{array}{c}\text { Pesquisa } \\
\text { Cualitativa }\end{array}$ & $\begin{array}{c}\text { Entrevista } \\
\text { Observación } \\
\text { participante y } \\
\text { notas de campo }\end{array}$ \\
\hline 6 & $\begin{array}{l}\text { A interação/atuação } \\
\text { da equipe do } \\
\text { programa de saúde } \\
\text { da família do Canal } \\
\text { Anil com a } \\
\text { população idosa } \\
\text { adscrita } \\
\end{array}$ & $\begin{array}{l}\text { Castro, MG; } \\
\text { Vargas, LA }\end{array}$ & $\begin{array}{l}\text { Physis: revista } \\
\text { de saúde } \\
\text { coletiva }\end{array}$ & Enfermero & 2005 & $\begin{array}{c}\text { Pesquisa } \\
\text { Cualitativa }\end{array}$ & $\begin{array}{l}\text { Observación } \\
\text { participante y } \\
\text { entrevista } \\
\text { semiestructu- } \\
\text { rada }\end{array}$ \\
\hline 7 & $\begin{array}{l}\text { A participação da } \\
\text { família no cuidado } \\
\text { ao prematuro } \\
\text { neonatal }\end{array}$ & $\begin{array}{c}\text { Gaíva, } \\
\text { MAM; } \\
\text { Scochi, CGS }\end{array}$ & $\begin{array}{c}\text { Revista } \\
\text { brasileira de } \\
\text { enferma-gem }\end{array}$ & Enfermero & 2005 & $\begin{array}{c}\text { Pesquisa } \\
\text { Cualitative }\end{array}$ & $\begin{array}{l}\text { Observación } \\
\text { particpante }\end{array}$ \\
\hline 8 & $\begin{array}{l}\text { Promoção da saúde } \\
\text { e qualidade de vida } \\
\text { entre mães de pré- } \\
\text { adolescentes: um } \\
\text { estudo etnográfico } \\
\text { enfocado em } \\
\text { Timbó/SC- Brasil }\end{array}$ & $\begin{array}{l}\text { Radunz, V; } \\
\text { Olson, J }\end{array}$ & $\begin{array}{c}\text { Revista latino } \\
\text { americana de } \\
\text { enfermagem }\end{array}$ & Enfermero & 2005 & $\begin{array}{c}\text { Pesquisa } \\
\text { Cualitativa }\end{array}$ & $\begin{array}{c}\text { Entrevista } \\
\text { semiestructura- } \\
\text { da, observación } \\
\text { participante, } \\
\text { diario de } \\
\text { campo, } \\
\text { etnograma y } \\
\text { fotografías }\end{array}$ \\
\hline 9 & $\begin{array}{l}\text { Promoción de salud } \\
\text { Y calidad de vida } \\
\text { em madres de } \\
\text { preadolescentes de } \\
\text { la comunidad de } \\
\text { Chiguayante-Chile- } \\
\text { Uma etnografia } \\
\text { enfocada }\end{array}$ & $\begin{array}{l}\text { Silva, JH; } \\
\text { Olson, J }\end{array}$ & $\begin{array}{c}\text { Revista latino } \\
\text { americana de } \\
\text { enfermagem }\end{array}$ & Enfermero & 2005 & $\begin{array}{c}\text { Pesquisa } \\
\text { Cualitativa }\end{array}$ & $\begin{array}{c}\text { Entrevista } \\
\text { semiestructura- } \\
\text { da, observación } \\
\text { participante, } \\
\text { diario de } \\
\text { campo, } \\
\text { etnograma y } \\
\text { fotografias }\end{array}$ \\
\hline 10 & $\begin{array}{l}\text { The meanings of } \\
\text { quality of life: } \\
\text { inbterpretative } \\
\text { analyses based on } \\
\text { experiences of } \\
\text { people in burns } \\
\text { rehabilitation }\end{array}$ & $\begin{array}{l}\text { Costa, MCS; } \\
\text { Rossi,LA; } \\
\text { Lopes, LM; } \\
\text { Cioffi, CL }\end{array}$ & $\begin{array}{c}\text { Revista latino } \\
\text { americana de } \\
\text { enfermagem }\end{array}$ & Enfermero & 2008 & $\begin{array}{c}\text { Pesquisa } \\
\text { Cualitativa }\end{array}$ & $\begin{array}{l}\text { Observación y } \\
\text { entrevista } \\
\text { semiestructu- } \\
\text { rada }\end{array}$ \\
\hline 11 & $\begin{array}{l}\text { Organização do } \\
\text { trabalho de uma } \\
\text { equipe de saúde no } \\
\text { atendimento ao } \\
\text { usuário em } \\
\text { situações de } \\
\text { urgência e } \\
\text { emergência }\end{array}$ & $\begin{array}{l}\text { Garlet, ER; } \\
\text { Lima, } \\
\text { MADS; } \\
\text { Santos, JLG; } \\
\text { Marques, } \\
\text { GQ }\end{array}$ & $\begin{array}{c}\text { Texto e } \\
\text { contexto } \\
\text { Enfermagem }\end{array}$ & Enfermero & 2009 & $\begin{array}{l}\text { Cualitativa } \\
\text { Estudio de } \\
\quad \text { caso }\end{array}$ & $\begin{array}{l}\text { Observación y } \\
\text { entrevista } \\
\text { semiestructura- } \\
\text { da }\end{array}$ \\
\hline
\end{tabular}




\begin{tabular}{|c|c|c|c|c|c|c|c|}
\hline 12 & $\begin{array}{l}\text { Moving drom } \\
\text { reclusion to partial } \\
\text { freedom: the } \\
\text { experience of } \\
\text { family caregivers } \\
\text { for disabled elderly } \\
\text { persons assisted in a } \\
\text { Day care center }\end{array}$ & $\begin{array}{l}\text { Bocchi, } \\
\text { SCM; Cano, } \\
\text { KCU; } \\
\text { Baltieri,L et } \\
\text { al. }\end{array}$ & $\begin{array}{c}\text { Ciência e } \\
\text { saúde coletiva }\end{array}$ & Enfermero & 2010 & $\begin{array}{c}\text { Cualitativo } \\
\text { Interaccio- } \\
\text { nismo } \\
\text { simbólico }\end{array}$ & $\begin{array}{l}\text { Observación y } \\
\text { entrevista }\end{array}$ \\
\hline
\end{tabular}

Se puede observar en la Tabla II que de los 12 estudios de investigación encontrados 11 fueron con enfoque cualitativo y 1, cuantitativo. Respecto al año de publicación, prevaleció 2005 con mayor enfoque, totalizando cinco artículos sobre la temática. El periódico que más manuscritos presentó sobre calidad de vida utilizando la metodología de observación fue la Revista Latino Americana de Enfermagem, con 6 artículos durante el tiempo de pesquisa. Todos los artículos tenían como autores por lo menos un enfermero. Todas las producciones fueron escritas por lo menos por un enfermero.

Los artículos que compusieron el corpus de análisis fueron categorizados de acuerdo con la metodologia utilizada, por ser esta el foco del trabajo: observación como técnica de colecta de datos, estando o no asociada a otra técnica. La observación y la entrevista presentaron mayor frecuencia: seis utilizaron la entrevista y observación participante como método de colecta de datos: dos utilizaron la observación participante, entrevista semiestructurada, diario de campo, etnografia y fotografia: una utilizó la observación libre y revisión de prontuários; una, entrevista, cuestionario y observación libre: una, entrevista, observación libre y apuntes de campo, y una utilizó la observación participante.

Se percibe que solo un artículo utilizó la observación como método de colecta de datos y que la mayoría utiliza la entrevista semiestructurada asociada a la técnica observacional totalizando, en este caso, diez de los artículos estudiados.

\section{DISCUSIÓN}

En la Tabla 1 se observó la exclusión de casi la mitad de los artículos científicos con el pasor de los años. Por lo tanto, se percibe que los investigadores enfermeros presentan ciertas dificultades en trabajar la metodología de la observación relacionada con la temática de calidad de vida.

La observación se caracteriza como una técnica de colecta de datos que consigue las informaciones y utiliza los sentidos en la obtención de determinados aspectos de la realidad, no consistiendo solo en ver y oir, sino fundamentalmente en observar hechos o fenómenos que se desea investigar ${ }^{(6)}$.

Por saber que calidad de vida es un constructo subjetivo, hay que tener en cuenta que muchas veces lo que se observa puede no ser sentido por los sujetos investigados. Así, autores corroboran la idea de que la calidad de vida es compleja, multifactorial, dinámica e incluye la opinión de que existen múltiples dimensiones involucradas, tales como: culturales, materiales, psicológicas, espirituales, emocionales y ambientales. En este contexto ideas y sentimientos personales también están involucrados con relación a todas estas dimensiones $^{(7)}$. Es importante subrayar que puede ser por este concepto y por la subjetividad de la temática que ocurre la dificultad de los enfermeros en utilizar la metodología de la observación en el tema calidad de vida. 
Se puede ver en la Tabla 2 que de los doce artículos identificados, dos fueron construidos por autores de áreas distintas, lo que puede llevar a ausencia de exploración de la interdisciplinaridad. Las pesquisas interdisciplinares son de fundamental importancia pues las disciplinas aisladamente no consiguen explicitar la totalidad. Por lo tanto, la interdisciplinaridad es una posibilidad de contribución a la nitidez y quizá para elucidar de manera más fidedigna un objeto que es común a varios profesionales ${ }^{(8)}$.

Concerniente a la metodología utilizada para la colecta de datos fueron establecidas dos categorias de análisis referentes a observación: observación participante y observación libre. La discusión que se inicia se basa en los doce artículos corpus de esta pesquisa.

\section{Calidad de vida y observación participante}

Al hablar de observación participante hay que considerar la participación real del investigador en la comunidad o grupo que está siendo investigado. En este sentido, ¿el investigador se incorpora al grupo confundiéndose con él? Tenemos así, un intento de disponer el observador y lo observado en un mismo lado.

Para discutir calidad de vida y observarla basada en esta metodología de colecta de datos, inicialmente tenemos que percibir el escenario cultural donde estos sujetos están insertados. Corroborando este énfasis hay que considerar que la calidad de vida se altera en la medida que las necesidades humanas no son totalmente satisfechas. En este constructo el investigador necesita reconocer que el estudio de calidad de vida y la observación de la misma representan un desafio ya que la importancia de la observación participante reside en el hecho de que se puede captar una variedad de situaciones o fenómenos que no podrían ser obtenidos por medio de la propia realidad, transmitiendo lo que de más fidedigno existe en la vida real ${ }^{(9)}$.

El enfermero, evaluando la calidad de vida por medio de la observación participante, puede medir que este es un tema y metodología dinámica donde existe la necesidad de contextualizar el ambiente para la búsqueda de lo real y de lo concreto. Por tanto, abordar calidad de vida por medio de la observación participante surge en la visualización de un mundo complejo, interconectado e interactivo.

Hay que considerar también que los artículos investigados y que utilizaron la observación participante en investigaciones sobre el tema calidad de vida, se unieron en su mayoría a otras técnicas de colecta entre lãs que sobresale la entrevista. Los estudios apuntan a que la unión de las dos técnicas permite obtener opiniones y percepciones más significativas presentando así hallazgos verdaderamente reales ${ }^{(10)}$.

\section{Calidad de vida y observación libre}

La observación libre, también llamada asistemática, se caracteriza por ser espontánea, informal y ocasional, consistiendo en recoger hechos y registrarlos sin que el investigador utilize medios técnicos especiales o necesite realizar preguntas directas. En este momento, es más utilizada en estudios exploratorios sin tener planeamiento y control elaborados ${ }^{(6)}$.

Evaluar calidad de vida por medio de la observación libre puede resultar en hechos no visualizados. Esto porque la calidad de vida implica aspectos subjetivos y objetivos y por eso muchos aspectos pueden quedar sin respuesta o explicación real. El profesional de salud al utilizar esta técnica de colecta de datos, en especial en la temática calidad de vida, 
generalmente se asegura en otra técnica que puede ayudar al estudio con informaciones más pertinentes sobre lo que se quiere investigar.

Ante todo lo expuesto queda claro que la utilización de la técnica dependerá del investigador, de su atención a los fenómenos que ocurren a su alrededor, de su perspicacia, discernimiento al observar, preparación y entrenamiento. Esto se debe al hecho de que todas las proyecciones observadas relacionadas con calidad de vida pueden transformarse rápidamente en reflejos directos en lo que es observado ${ }^{(11)}$.

En este sentido, se percibe la necesidad de utilización de otra técnica de colecta de datos, junto a la observación libre, ya que la suma de las dos técnicas puede contribuir al descubrimiento más sostenible científicamente de lo que se quiere estudiar/investigar.

Así, evaluar el impacto de la calidad de vida por medio de la observación libre se convierte em un desafio por la complejidad en cuantificar factores subjetivos. Por lo expuesto, se percibe que calidad de vida no puede ser tomada y observada como un concepto general, sino entendida dentro de la experiencia cotidiana y personal de cada uno de los sujetos ${ }^{(12)}$.

\section{CONSIDERACIONES FINALES}

Por la presente investigación de revisión integrativa se puede percibir la necesidad emergente de más estudios en enfermería sobre calidad de vida con utilización de la técnica de observación considerando que la temática es ampliamente discutida en la actualidad en distintos ámbitos. Además, existe también la preocupación sobre la realización de trabajos interdisciplinares utilizando la herramienta de la observación como colecta de datos, lo que no fue observado en los estudios encontrados.

Se cree que se logro el objetivo trazado anteriormente, pero con un número pequeño de trabajos que se direccionan al objetivo. De esta manera, esta revisión ha confirmado una vez más la dificultad de medir calidad de vida, por ser compleja y dinamica además de poseer una naturaleza multifactorial que puede interferir en lo que está siendo observado.

Además, esta revisión integrativa puede instigar investigadores enfermeros que abordan la temática de calidad de vida en la enseñanza, en la investigación o en la extensión para que trabajen con la temática en un abordaje observacional, considerando el pequeño número de trabajos publicados.

De este modo, considerando la subjetividad y objetividad de la temática se concluye que toda la experiencia cotidiana, sea en aspectos materiales de calidad de vida o en su dimensión subjetiva, tiene en la observación un instrumento de relieve que puede contribuir, con otros métodos de colecta de datos, a una percepción integral de lo que se quiere investigar.

\section{REFERÊNCIAS}

1. Lentz RA, Costenaro RGS, Gonçalvez LHT, Nassar SN. O profissional de enfermagem e a qualidade de vida: uma abordagem fundamentada nas dimensões propostas por Flanagan. Rev Latino Enferm Ribeirão Preto. 2000 Ago; 8(4):7-14.

2. Zamberlan C, Calvetti A, Deisvaldi J, Siqueira HCH. Qualidade de vida, salud y enfermería en la perspectiva ecosistémica. Enfermería Global. 2010;10:1-7.

3. Pope C, Mays N. Métodos observacionais. In: Pope C, Mays N. Pesquisa Qualitativa na atenção à saúde. Porto Alegre (RS): Artmed; 2004. p. 46-55. 
4. Menezes KDS, Silveira RCCP, Galvão CM. Revisão integrativa: método de pesquisa para a incorporação de evidências na saúde e na enfermagem. Texto Contexto Enferm. 2008; 17(4):758-64.

5. Cooper $\mathrm{H}$. The integrative research review: a systematic approach. NY: Sage Pub; 1984.

6. Marconi MA, Lakatos EM. Fundamentos da metodologia científica. São Paulo: Atlas; 2003.

7. Radunz V, Olson J. Promoção da saúde e qualidade de vida entre mães de préadolescentes: um estudo etnográfico enfocado em Timbó/SC, Brasil. Rev Lat Am Enfermagem. 2005 Nov-Dez; 13(2):1-8.

8. Santos SSC, Lunardi VL, Herdmann AL, Caloni H. Interdisciplinaridade: a pesquisa como eixo de formação/profissionalização na saúde/enfermagem. Revista Didática Sistêmica. 2007 Jan-Jun; 5:13-22.

9. Minayo MCS. Pesquisa social: teoria, método e criatividade. Rio de Janeiro: Vozes; 1998.

10. Andrade GRB, Vaitsman J. Apoio social e redes: conectando solidariedade e saúde. Ciência e Saúde Coletiva [internet]. 2002. [Acesso em: 5 jan 2011];7(4). Disponível em: http://www.scielo.br.

11. Castro MR, Vargas LA. A interação/atuação da equipe do programa de saúde da família do Canal do Anil com a população idosa. Adscrita. Physis: Revista de Saúde Coletiva [internet]. 2005. [acesso em 5 jan 2011];15(2). Disponível em: http://www.scielo.br.

12. Souza RA, Carvalho AM. Programa de saúde da família e qualidade de vida: um olhar da psicologia. Revista Estudos de psicologia, 2003 Set-Dez; 8(3):515-23. 